\title{
Statistical Applications for SNPs Analysis
}

\author{
Woosung Yang $^{1 *}$, Jun Nakaya ${ }^{1}$ \\ ${ }^{1}$ Clinical Genome Informatics Center, Kobe University, \\ 7-5-2 Kusunokicho, Chuo-ku, Kobe-city, Hyogo, 650-0017, Japan \\ *E-mail: wyang@med.kobe-u.ac.jp
}

(Received July 24, 2006; accepted September 28, 2006; published online October 26, 2006)

\begin{abstract}
A subset of single nucleotide polymorphisms (SNPs) can be used to capture the majority of the information of genotype-phenotype association studies. The primary issue that we should discuss in SNPs analysis is how one can select a particular subset of SNPs while maximizing the power of detecting a significant association. From this point of view, we reviewed some statistical tools for SNPs analysis by focusing in this paper on the Hardy-Weinberg equilibrium, case-control association study, and haplotype estimation and association. We also paid special attention to the issue of how we can determine the required sample size by using the sequential test. With regard to the statistical tools for SNPs analysis, we reviewed the basic concepts of each method and its applications.
\end{abstract}

Key Words: Hardy-Weinberg equilibrium, SNPs, Association study, Linkage disequilibrium, Sequential test

Area of Interest: Genome Wide Experimental Data Analyses

\section{Introduction}

For many diseases a body of knowledge regarding the pathology of the disease progression has already been made available. For example many autoimmune diseases, such as Rheumatoid Arthritis (RA) and Multiple-Sclerosis (MS), are known to involve inflammation at the active site. Thus biologically active components of the inflammatory system would make good candidate genes. In general it is useful to have an understanding of the clinical and biological aspects of the disease that we are interested in, as it will help guide us in our search for disease susceptibility loci. One may also want to choose genes that have a demonstrated association with a disease related to the disease of interest, as many complex diseases are likely to share components that influence susceptibility. Another approach to determining candidate genes is the use of animal models. There are a number of diseases for which well-defined animal models exist in other organisms. Because human have a shared evolutionary history with these organisms, it is possible that genes linked to 
disease susceptibility in such model organisms play a role in the etiology of the disease in humans. After such a gene has been identified within the model organism, it is possible to use BLAST searches to find candidates for homologous regions within the human genome to test for association.

The detection of susceptibility genes for complex traits has attracted much interest in human genetics. There is increasing interest in using single nucleotide polymorphisms (SNPs) for detailed candidate gene [36] and genome-wide [33][7] association studies of common diseases. The study of genome-wide association in complex traits requires a large sample size, because the expected effect of each gene is small and extremely low significance levels need to be adopted [32][30]. In addition to the large sample size required, however, the cost of genotyping is frequently one of the most important considerations. If the total budget for genotyping is limited, it is very important to choose the optimal study design for the budget available [1][16].

Linkage disequilibrium (LD) between proximal sites suggests that it is possible to detect disease association by using a relatively sparse collection of SNPs that are in LD with a disease susceptibility allele. The question is how to select the subset of SNPs that are informative for disease association studies. In other words, given that resources are limited, how does one select a subset of SNPs to be genotyped in order to maximize the ability to detect disease association? Kruglyak[25] concluded that a useful level of LD is unlikely to extend beyond an average of $3 \mathrm{~kb}$, so that approximately 50,000 SNPs would be required for a genome-wide association study.

Sequential analysis is the branch of statistics concerned with investigations in which the decision whether or not to stop at any stage depends on the observations previously made. In the present paper we introduce a number of statistical tools for SNPs analysis.

\section{Hardy-Weinberg Equilibrium}

In the absence of migration, mutation, natural selection, and assortative mating, genotype frequencies at any locus are a simple function of allele frequencies. This phenomenon, now termed "Hardy-Weinberg equilibrium" (HWE), was first described in the early part of the twentieth century [19][41]. The ordinal descriptions under HWE are important landmarks in the history of population genetics [8], and it is now common practice to check whether observed genotypes conform to Hardy-Weinberg expectation. These expectations appear to hold for most human population structures or, in samples of affected individuals, an association between the marker and disease susceptibility.

The HWE is an expression of the notion of a population in "genetic equilibrium" and is a basic principle of population genetics. When the Hardy-Weinberg assumptions are not met, this can cause deviations from expectation, but depending upon which assumption is not met, such deviations may or may not be statistically detectable. Deviations can be caused by the Wahlund effect, inbreeding, assortative mating, selection, or genetic drift. Assortative mating will only change the genotype frequencies of those genes that are desired. Genetic drift is particularly active in small population sizes. Deviations caused by selection, however, often require a significant selection coefficient in order to be detected, which is why the test for deviations from Hardy-Weinberg proportions is considered a weak test for selection.

A more statistical description for the HWE is that the alleles for the next generation for any given assorted individual are chosen independently. Consider two alleles, $A$ and $a$, with frequencies $p$ and $q$, respectively, in the population, then the different ways to form new genotypes can be derived by using a Punnett square, where the size of each cell is proportional to the fraction of each genotype in the next generation: 
The final three possible genotype frequencies in the offspring, if the alleles are assorted independently, become

$$
\begin{aligned}
& f(A A)=p^{2} \\
& f(A a)=2 p q \\
& f(a a)=q^{2}
\end{aligned}
$$

This is normally achieved in one generation, except when a population is created by bringing together males and females with different allele frequencies, in which case, equilibrium is reached in two generations. Where the gene is sex-linked, the heterogametic sex (e.g. males in humans) has only one copy of the gene and is effectively haploid for that gene. So the genotype frequency at equilibrium is therefore $p$ and $q$ for the heterogametic sex but $p^{2}, 2 p q$ and $p^{2}$ for the homogametic sex. For example in humans, red-green color-blindness is caused by an X-linked recessive allele. The frequency in males is about $1 / 12$, whereas it affects about $1 / 250$ females. If a population is brought together with males and females having different allele frequencies, then the allele frequency of the male population follows that of the female population because each receives its $\mathrm{X}$ chromosome from its mother. The population converges on equilibrium, within about six generations maximum.

The HWE may be generalized to more than two alleles. Consider an extra allele frequency, $r$. The two-allele case is the binomial expansion of $(p+q)^{2}$, and thus the three-allele case is

$$
(p+q+r)^{2}=p^{2}+q^{2}+r^{2}+2 p q+2 p r+2 q r
$$

More generally, consider the alleles $A_{1}, A_{2} \cdots, A_{i}$ given by the allele frequencies $p_{1}, p_{2}, \cdots p_{i}$,

$$
\left(p_{1}+p_{2}+\cdots+p_{i}\right)^{2}
$$

giving for all homozygotes $f\left(A_{i} A_{i}\right)=p_{i}^{2}$, and for all heterozygotes $f\left(A_{i} A_{j}\right)=2 p_{i} p_{j}$, where $i \neq j$. The HWE may also be generalized to polyploid systems, that is, to populations having more than two copies of each chromosome. Consider again only two alleles. The diploid case is the binomial expansion of $(p+q)^{2}$ and therefore the polyploid case is the binomial expansion of $(p+q)^{c}$, where $c$ is the ploidy, for example with tetraploid, $c=4$,

$$
\begin{aligned}
& f(\text { AAAA })=p^{4} \\
& f(\text { AAAa })=4 p^{3} q \\
& f(\text { AAaa })=6 p^{2} q^{2} \\
& f(\text { Aaaa })=4 p q^{3} \\
& f(\text { aaaa })=q^{4}
\end{aligned}
$$

The complete generalized formula is the multinomial expansion of $\left(p_{1}+p_{2}+\cdots p_{i}\right)^{n}$, 


$$
\left(p_{1}+p_{2}+\cdots+p_{i}\right)^{n}=\sum_{k_{1}, k_{2}, \cdots k_{n}}\left(\begin{array}{c}
n \\
k_{1}, k_{2}, \cdots, k_{n}
\end{array}\right) p_{1}^{k_{1}}, p_{2}^{k_{2}}, \cdots p_{n}^{k_{n}}
$$

The HWE may be applied in two ways, either a population is assumed to be in Hardy-Weinberg proportions, in which the genotype frequencies can be calculated, or if the genotype frequencies of all three genotypes are known, they can be tested for deviations that are statistically significant. Suppose that the phenotypes of $A A$ and $A a$ are indistinguishable, i.e., that there is complete dominance. Assuming that the HWE applies to the population, then $q$ can still be calculated from $f(a a)$,

$$
q=\sqrt{f(a a)}
$$

and $p$ can be calculated from $q$. In this way, an estimate of $f(A A)$ and $f(A a)$ can be derived from $p^{2}$ and $2 p q$, respectively. Note, however, that such a population cannot be tested for equilibrium by using the significance tests below, because it is assumed a priori. Pearson's chi-square test is generally used to test for deviation from the HWE, with the observed genotype frequencies obtained from the data and the expected genotype frequencies obtained using the HWE. For systems where there are large numbers of alleles, this may result in data with many empty possible genotypes and low genotype counts, because there are often not enough individuals present in the sample to adequately represent all possible genotype classes. If this is the case, then the asymptotic assumption of the chi-square distribution, will no longer hold, and it may be necessary to use a form of Fisher's exact test, which requires a computer to solve.

The principles and procedures used for testing HWE are well established [26][18][20][42], but the lack of a publicly available, efficient, and reliable implementation for exact tests had led many scientists to rely on asymptotic tests that can perform poorly with realistic sample sizes. Janis et. al. [23] proposed efficient implementations of the exact test for HWE, which are suitable for use in large-scale studies of SNP data, even when hundreds of thousands of markers are examined.

\section{Case-Control Association Test}

With the completion of the Human Genome Project, there is a lot of information readily available about the occurrence and frequency of polymorphisms throughout the human genome. This information is invaluable and should be used to help in the selection of SNPs for genotyping. Ideally one should choose SNPs that have a high degree of polymorphism (i.e., minor allele frequency $>10 \%$ ) in the population. We can improve our chances of detecting an association if we choose exonic SNPs that result in non-synonymous coding changes or are located in known regulatory regions. This information can be obtained from the bioinformatics web sites. Because resources are often limited (in terms of money available for genotyping), it is often beneficial to perform studies in a two-stage approach by using a subset of samples to identify those SNPs that provide the maximum amount of information about the structure of polymorphisms within the region of interest. Ideally, one should genotype around a hundred controls to characterize haplotype tagging SNPs (htSNPs); however, one may wish to genotype a sampling of cases as well, and carry out some preliminary tests of association to help in the selection of htSNPs.

Once we have genotyped the subset, we should test all loci for the Hardy-Weinberg Equilibrium (HWE). If loci are found to be out of HWE then it is evidence of selection, or more likely genotyping error, and we should check the genotyping and repeat it, if required. 
Association analysis may be regarded as a test for the presence of a difference in allele frequency between case and controls. A difference does not necessarily imply causality in disease, as many factors, including population history and ethnic make-up may yield this effect. In a well-designed study, however, evidence of association provides a flag for further study.

Association testing for case-control data is often carried out using general (non-genetic) statistical software packages, such as SAS or S-PLUS. In this setting two factors appear, disease status and genotype. The levels for disease status are case and control. The levels for genotype are various genotypes observed among either the case or controls. Because cases often differ from controls in the overabundance of one or two genotypes, it is desirable to implement a test that is sensitive to such departures.

A $\chi^{2}$ test is applied to a contingency table, in which the case/control status is tabulated by frequencies of either genotype or alleles. The test takes the usual form

$$
\chi^{2}=\sum \frac{(O b s-\operatorname{Exp})^{2}}{\operatorname{Exp}}
$$

where $O b s$ and Exp are the observed and expected frequencies, respectively; and the sum is taken over all cells in the table. The number of degrees of freedom is $(r-1)(c-1)$, where $r$ is the number of rows and $c$ is the number of columns in the table.

The Odds-Ratio is a widely used statistic for comparing the frequency of exposure to risk factors in epidemiological studies. Most people will be familiar with odds from betting, and odds ratios are simply comparing the retrospective/posterior odds of exposure to a given risk factor in two groups of individuals. There are special methods that allow the calculation of multiple levels of odds-ratio. Calculating the odds-ratio for a set of data is fairly straight-forward, we need to construct a contingency table in the same manner as we do for $\chi^{2}$ calculations; although in this instance, we should always place the cases in the first row and the risk factor in the first column [44].

It has long been appreciated [6] that misclassification errors can significantly affect the results of statistical tests of association. In the field of genetics, researchers may observe misclassification errors in phenotype and/or genotype. A major question in the field of statistical genetics is: how does one "deal" with such errors when performing tests of genetics association? Much work has been done to address the general question of errors in statistical tests, and recently some work has been done to address the specific question of errors in genetic tests of association.

Breslow and Day [3] attributed the first statistical work on errors in the association tests applied to contingency tables to Bross [4]. In this work, Bross focused on the $\chi^{2}$ test of independence applied to $2 \times 2$ contingency tables. Bross's findings were: assuming that the error procedure is independent of case/control status, there is no change in the level of significance; power for the $\chi^{2}$ test is reduced; and, there is a bias in point estimates of the population frequency parameters.

Monte and Anderson [28] synthesized the work of Mitra [27] and Bross. They proved that the power of the $\chi^{2}$ test with no error is always greater than or equal to the power of the test when errors are present and ignored. Tenenbein [37][38] presented a procedure that used perfect classification on a sub-sample of data to estimate the classification rates for all categories and also provided asymptotically unbiased estimates of population parameters (e.g., genotype frequencies, proportion of cases and controls). He called this procedure a "double-sampling" procedure, because some observations are sampled twice--once with a perfect classifier, and once with a fallible and usually less expensive classifier. Chen [5] incorporated Tenenbein's work into a log linear model. 
Hochiberg [21], also using Tenenbein's work, developed both a least squares approach and a combined maximum likelihood and least squares approach for analyzing multi-dimensional cross-classified data subject to error.

Gustafson et al. [17] used a Bayes analysis to adjust for uncertainty in misclassification probabilities as prior information and thereby improve estimates of the odd-ratio in case-control data. In related work for the $2 \times 3 \quad \chi^{2}$ test of genetic association with a di-allelic locus, Rice and Holmans [31] incorporated genotyping misclassification mistakes into the calculation of confidence interval estimates for parameters such as genotypic relative risk.

Gordon and Ott [15] considered the analysis of genetic data in the presence of genotyping error. They confirmed that: (i) there is no increase in type I error for certain tests of genetic association; (ii) point estimates of the frequencies of SNP alleles are biased in the presence of errors; and (iii) errors lead to a loss in power to detect an association between a disease and a locus. Recently, Gordon et al. [13][14] produced a quantification of the loss in power for case-control studies of genetic association due to genotyping errors.

A critical but unanswered question is how one can use information about misclassification to improve statistical power for genetic tests of association using case-control data.

There are several problems associated with carrying out genetic association studies; and perhaps the most overbearing issue is the availability of resources, in terms of funding for carrying out the study, and the availability of subjects to study. Another important issue is the problem of multiple testing that arises when a large number of loci are tested for association with a disease. The above approach helps to minimize all of these features simultaneously by minimizing the number of polymorphisms that need to be genotyped and tested for association; however, you can still end up carrying out a large number of tests that require statistical correction if false positive-associations are to be controlled for.

The method described above is a two-step approach which seeks to minimize the amount of genotyping work that is required to test for association; however, it is not the only approach available. There are a number of alternative methods for carrying out the two steps involved. One approach is to test all variants in a subset of individuals for disease association, correct the p-values, and take those that are significant after multiple testing on to the second stage. To help overcome this problem, a number of different two-stage approaches have been proposed that test for association in a subset of individuals, correct for the multiple testing that has been done, and identify the remaining samples for those markers that displayed significant association [39].

Generally, power-calculations are performed when grant-proposals are made to ensure that the study will have sufficient power to detect a given effect. In order to calculate this, however, we need to decide on a few parameters first. Initially, we must decide on the power we wish our study to have (most studies hope to attain power of at least $80 \%$ ) as well as the significance level we wish to attain. In addition, we need to know the size of the effect that we are hoping to see; for example, does exposure to a given risk factor increase our odds of developing a disease by two-fold, three-fold, or higher. Note that the larger the effect size we are hoping to detect, the smaller the sample size required.

Once we have decided on these parameters, it is simply a case of plugging the numbers into the formula shown below.

One should not be tempted to use power as an explanation for any failure to detect association [22][12]. Do not ever use statements such as "Failure to detect association is due to the low power of this data set." For a full exposition of this line of reasoning, please read [12]. Statements regarding power should be made in the methods section along the lines of "It was calculated that a sample size of $\mathrm{X}$ was required to obtain power $\mathrm{Y}$ to detect an effect of size $\mathrm{Z}$ or greater." 
As well as the traditional $\chi^{2}$ and Odds-Ratios methods of testing for association, there are a number of other tests available. Mathematical details of how to calculate the various statistics are not provided; readers are instead referred to the original articles, which are referenced below.

- The Hardy-Weinberg Disequilibrium test

A number of authors [10][29][24] have proposed quantifying the difference in Hardy-Weinberg equilibrium between cases and controls as a means of testing for disease association.

\section{- Trend Test (Additivity)}

If cases or controls are not in Hardy-Weinberg equilibrium, then it has been shown [34] that Armitage's trend test [2] should be used to test for association at the genotypic level. These methods have been extended to account for population heterogeneity and utilize Bayesian methods to correct for multiple testing when large numbers of SNPs are being tested [9].

\section{- Weighted Average}

Recently Song and Elston [35] proposed the weighted average method of testing for association using differences in the above test statistics between cases and controls. This test combines the strong point of both of the above tests while overcoming the weakness.

\section{Haplotype Estimation and Association}

When we are investigating a disease association, it is important to bear in mind that the mutations we are seeking to detect did not occur in isolation, but on a chromosome that already had a number of different mutations within the region. Thus, at the point in time when the mutation arose, it was in complete linkage disequilibrium (LD) with all other mutations on that chromosome and was inherited together with a number of those mutations. Over time, the LD will decline between the disease mutation and markers that are further away as a consequence of recombination, but it will remain in LD with those markers that are nearby, and it may therefore be possible to define a risk modifying haplotype in a region of the genome surrounding the disease mutation. Once we have identified multiple single-point associations within a region, we can, by defining and testing haplotypes, test for disease associations.

How we estimate haplotypes depends upon the study that we are carrying out. If we have three generational families, then it is possible to infer the phase of an individuals genotypes (i.e., upon which of the two chromosomes a series of markers lie for a given individual). But if we have case-control data or two generational families, then we are not able to infer phase (and therefore haplotypes), and must instead resort to estimation. There are a number of different methods for estimating haplotypes, such as the EM algorithm or Bayesian methods. Described below are several different programs that will infer haplotypes for data. Which method we use depends very much on the number and type of markers for which have data, although it is often good practice to try a couple of different methods to ensure we are getting the same answer from different methods (this will improve the confidence with which we can claim to have estimated haplotypes accurately).

There are a number of different software packages available for inferring haplotypes in both phased and unphased data, as well as for tagging SNPs.

- QTLHAPLO: Estimation of haplotype frequencies and quantitative trait parameters using the EM algorithm. 
- EH+: A faster implementation of the original EH (Estimate Haplotypes) program using the EM algorithm. Can handle multi-allelic data.

- SNPHAP: A fast program for inferring SNP haplotypes in data using the EM algorithm.

- PHASE: Bayesian estimation of haplotype frequencies in unphased data. Can handle multi-allelic data.

- Fugue: Haplotype estimation for SNPs as used for the investigation of haplotypes on chromosome 21 . Includes the ability to test estimate haplotype frequencies in cases and controls independently and test for association.

- Genehunter: A linkage analysis program that reports haplotypes. Can handle multi-allelic loci, but assumes linkage equilibrium between loci (not always true when looking at tightly-linked loci).

- Merlin: A linkage analysis program that reports haplotypes. Can handle multi-allelic loci, but assumes linkage equilibrium between loci (not always true when looking at tightly-linked loci).

When SNPs are in linkage disequilibrium with each other, there is a degree of redundancy in the information contained within a haplotype (proportional to the strength of LD). This feature can be exploited, and the diversity within a region can be captured, by typing a subset of markers. This means that the number of markers (and in turn the total cost of characterizing a region) can be reduced.

When inferring haplotypes within a region of reasonable linkage disequilibrium, a large proportion of the diversity of haplotypes can be covered by a small fraction of the haplotypes. That is to say, the diversity of haplotypes is accounted for by a few common haplotypes and many rare ones. The common haplotypes will share a number of SNPs in common with each other, whilst the rarer haplotypes will be characterized by carrying the rarer alleles at certain loci. Thus, the theory is that we can capture the majority of the diversity within a region by typing those SNPs that allow us to cover the most diversity. If we assess the diversity across all loci within a region in a subset of samples, we can then determine which loci within that region capture the majority of the diversity and then type them in remaining samples, thus reducing the overall cost of the study. Essentially, we are removing redundant information from the study. Obviously underpinning this whole theory is the Common Disease/Common Variant hypothesis which proposes that the underlying genetic components of common complex diseases are themselves common in frequency and distribution across a population; so the approach of using htSNPs when investigating regions for association to a particular disease should only be applied for those disease loci that are suspected to be common in occurrence. It is unlikely to be successful at picking up associations with disease loci that are rare.

There are two common approaches to tagging SNPs, those that derive tag SNPs from inferred haplotypes (so called block-based tagging), and those that are independent of the haplotype structure within a region (generally known as entropy based).

Block-based tagging requires that haplotype "blocks" first be inferred. In the majority of cases when we are investigating association within a candidate gene, we are likely to start off with a large number of potential SNPs to choose from, and using various measures of linkage disequilibrium and inferred haplotypes it is possible to define 'haplotype blocks' of markers that are in strong LD with each other, but not with those in other blocks. The exact definition of a haplotype block is open to interpretation, and there are a number of different methods for choosing your haplotype blocks [11]. The alternative to block-based tagging is known as entropy based, and rather than define haplotypes, the aim is to capture the most information across the region. Entropy is simply a measure of disorder. 


\section{Determining Required Sample Size}

In recent years, analyses of linkage disequilibrium (LD) are commonly performed to localize genes underlying complex traits. One major difficulty of studying the genetic background is that to reliably detect small effect size, large number of samples need to be recruited, phenotyped, and genotyped. All of which leads to more expensive study designs in terms of time and money. Therefore, it is important to find optimal conditions under the restricted cost and time conditions.

Generally, we used certain best test methods for determining a best critical region for testing a simple hypothesis against an alternative simple hypothesis. Let us now suppose that the sample size $n$ is not fixed in advance. In fact, let the sample size be a random variable $N$ with sample space $\{n: n=1,2,3, \cdots\}$. An interesting procedure for testing the simple hypothesis $H_{0}: \theta=\theta^{\prime}$ against the simple hypothesis $H_{1}: \theta=\theta^{\prime \prime}$ is the following. Let $k_{0}$ and $k_{1}$ be two positive constants with $k_{0}<k_{1}$. Observe the independent outcomes $X_{1}, X_{2}, X_{3}, \cdots$ in sequence, say $x_{1}, x_{2}, x_{3}, \cdots$, and compute

$$
\frac{L\left(\theta^{\prime}, 1\right)}{L\left(\theta^{\prime \prime}, 1\right)}, \frac{L\left(\theta^{\prime}, 2\right)}{L\left(\theta^{\prime \prime}, 2\right)}, \frac{L\left(\theta^{\prime}, 3\right)}{L\left(\theta^{\prime \prime}, 3\right)}, \cdots
$$

where $L(\theta, n)$ is the joint probability distribution function of $X_{1}, X_{2}, \cdots X_{n}$.

The hypothesis $H_{0}: \theta=\theta^{\prime}$ is rejected (and $H_{1}: \theta=\theta^{\prime \prime}$ is accepted) if and only if there exists a positive integer $n$ such that $\left(x_{1}, x_{2}, \cdots, x_{n}\right)$ belong to the set

$$
C_{n}=\left\{\left(x_{1}, x_{2}, \cdots, x_{n}\right) ; k_{0}<\frac{L\left(\theta^{\prime}, j\right)}{L\left(\theta^{\prime \prime}, j\right)}<k_{1}, j=1, \cdots, n-1, \text { and } \frac{L\left(\theta^{\prime}, n\right)}{L\left(\theta^{\prime \prime}, n\right)} \leq k_{0}\right\}
$$

On the other hand, the hypothesis $H_{0}: \theta=\theta^{\prime}$ is accepted (and $H_{1}: \theta=\theta^{\prime \prime}$ is rejected) if and only if there exists a positive integer $n$ such that $\left(x_{1}, x_{2}, \cdots, x_{n}\right)$ belong to the set

$$
B_{n}=\left\{\left(x_{1}, x_{2}, \cdots, x_{n}\right) ; k_{0}<\frac{L\left(\theta^{\prime}, j\right)}{L\left(\theta^{\prime \prime}, j\right)}<k_{1}, j=1, \cdots, n-1, \text { and } \frac{L\left(\theta^{\prime}, n\right)}{L\left(\theta^{\prime \prime}, n\right)} \geq k_{1}\right\}
$$

That is, we continue with sample observation as long as

$$
k_{0}<\frac{L\left(\theta^{\prime}, n\right)}{L\left(\theta^{\prime \prime}, n\right)}<k_{1}
$$

A test of this kind is called Wald's sequential probability ratio test [40]. Now, frequently, inequality equation (1) can be conveniently expressed in an equivalent form

$$
c_{0}(n)<u\left(x_{1}, x_{2}, \cdots, x_{n}\right)<c_{1}(0),
$$

where $u\left(X_{1}, X_{2}, \cdots, X_{n}\right)$ is a statistic and $c_{0}(n)$ and $c_{1}(n)$ depend on the constant $k_{0}, k_{1}, \theta^{\prime}, \theta^{\prime \prime}$, and on $n$. Then the observations are stopped and a decision is reached as soon as 


$$
u\left(x_{1}, x_{2}, \cdots, x_{n}\right) \leq c_{0}(n) \quad \text { or } \quad u\left(x_{1}, x_{2}, \cdots, x_{n}\right) \geq c_{1}(n)
$$

At this point, we shall denote the power of the test when $H_{0}$ is true by the symbol $\alpha$ and the power of the test when $H_{1}$ is true by the symbol $1-\beta$. Thus, $\alpha$ is the probability of committing a type I error (the rejection of $H_{0}$ when $H_{0}$ is true), and $\beta$ is the probability of committing a type II error (the acceptance of $H_{0}$ when $H_{0}$ is false). With the sets $C_{n}$ and $B_{n}$ as previously defined, and with random variables of the continuous type, we then have

$$
\frac{\alpha}{1-\beta} \leq k_{0}, \quad k_{1} \leq \frac{1-\alpha}{\beta}
$$

where $\beta$ is not equal to zero or 1 .

However, in a case-control study, usually there are only a limited number of observations per subject, and there are far more controls for which such material can be analyzed than cases. Therefore, it may be desirable to compensate for the loss of statistical power by comparing each case with more than one control.

Let $X_{i}$ be the observation for the $i$-th case, $Y_{i j}$ the observation for the $j$-th control $(j=1,2, \ldots, k)$ matched to the $i$-th case, $Y_{i}$ mean over the $k$-th controls matched to the $i$-th case, and $D_{i}=x_{i}-Y_{i}$ for $i=1,2, \ldots$. Further, let $X_{i} \square N\left(\mu_{x}, \sigma^{2}\right)$ and $Y_{i j} \square N\left(\mu_{y}, \sigma^{2}\right)$, then $D_{i} \square N\left(\mu, \sigma_{K}^{2}\right) \quad$ where

$$
\mu=\mu_{x}-\mu_{y}, \quad \sigma_{K}^{2}=\sigma^{2}(k+1) / k
$$

The parameter $\theta_{k}$, as a measure for the standardized difference, is defined as $\mu / \sigma_{k}$, where $\sigma_{k}=\sigma_{1} \sqrt{(k+1) / 2 k}\left(\sigma_{1}^{2}=2 \sigma^{2}\right)$. The null hypothesis to be tested is formulated as $H_{0}: \theta_{k}=0$ and the two-sided alternative hypothesis $H_{1}:\left|\theta_{K}\right| \geq \theta_{R}$, where $\theta_{R}$ is the minimum standardized difference. Tests are performed with a two-sided type I error $\alpha$ and a type II error $\beta$.

The test statistic for Wald's sequential probability ratio test (SPRT) is based on the likelihood ratio for $n$ data sets processed up to a given point. Rather than based on a likelihood ratio, Whitehead's procedure [43] is based on a profile log-likelihood function, considering $\sigma_{1}^{2}$ as a nuisance parameter. The parameter $\sigma_{1}^{2}$ is the variance of the difference between a case and a control observation. The test statistics are $Z$ (the efficient score for $\theta_{k}$ ) and $V$ (Fishers information contained in $Z$ ). For $k$ controls matched per case, the extended test statistics for the two-sided sequential t-test is formulated as

$$
Z=\sum_{i=1}^{n} d_{i} \sqrt{\left(\frac{2 k}{k+1} \frac{n}{\sum_{i=1}^{n} d_{i}^{2}}\right)}, V=n \frac{2 k}{k+1}-\frac{Z^{2}}{2 n}
$$

where $d_{i}$ is the difference between the observation for the case and control. For comparison, we 
considered an open double sequential test, which is a combination of two one-sided tests leading to stopping boundaries of the form

$$
\begin{array}{ll}
Z=a+b V\left(=u_{w 1}\right) & Z=-a+b V\left(=u_{w 2}\right) \\
Z=a-b V\left(=l_{w 2}\right) & Z=-a-b V\left(=l_{w 1}\right),
\end{array}
$$

where $u_{w 1}, u_{w 2}, l_{w 2}, l_{w 1}$ are the critical boundaries of the test. The slopes $\pm b$ and intercepts $\pm a$ of the boundaries are functions of the error probabilities $\alpha$ and $\beta$, as well as of the choice of a minimum standardized difference, $\theta_{R}$, which defines the alternative hypothesis $H_{1}:\left|\theta_{k}\right|=\theta_{R}$.

$$
\begin{gathered}
b=\frac{\delta}{\ln \left(\frac{1-\beta}{\beta}\right)+\ln \left(\frac{1-\alpha}{\alpha}\right)} \ln \left(\frac{1-\alpha}{\alpha}\right) \\
a=\frac{\ln \left(\frac{1-\beta}{\beta}\right)+\ln \left(\frac{1-\alpha}{\alpha}\right)}{2 \delta},
\end{gathered}
$$

where $\delta$ is the mean of the differences between the observations for the case and control. The sequential test following Whitehead's approach is presented in the form of a graph, plotting $Z$ against $V$ (see Figure 1). The testing process continues as long as the sample path formed by successive $Z$-values plotted against corresponding $V$-values remains between the boundaries $u_{w_{1}}$ and $u_{w_{2}}$ or between $l_{w_{1}}$ and $l_{w_{2}}$. The sampling is stopped and $H_{0}$ rejected when the sample path crosses $u_{w_{1}}$ or $l_{w_{1}}$. The test is stopped with acceptance of $H_{0}$ when the sample path crosses $u_{w_{2}}$ or $l_{w_{2}}$. The test is also stopped with the acceptance of $H_{0}$ when the sample path has crossed the first paths of both $u_{w_{2}}$ and $l_{w_{2}}$.

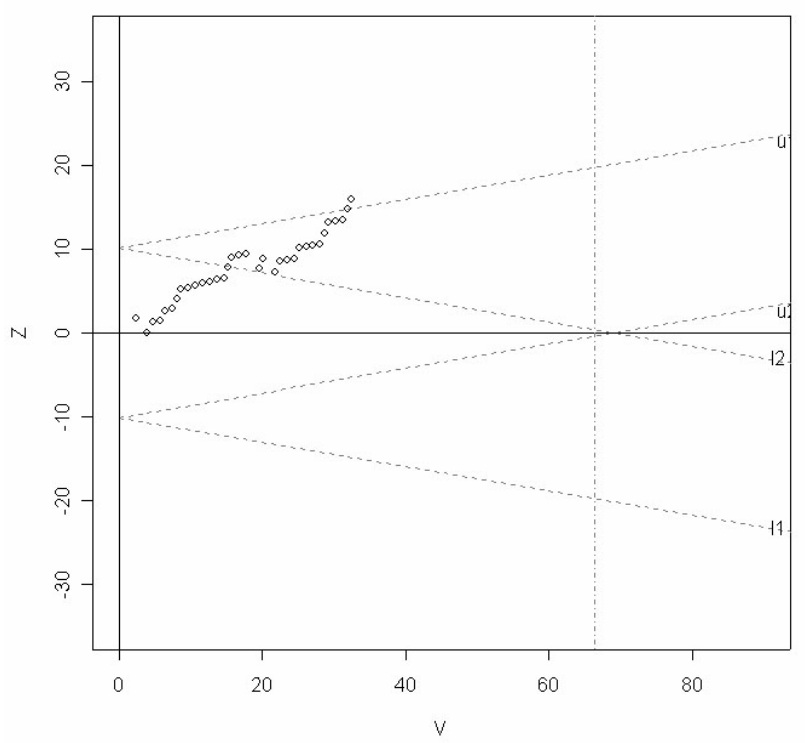

Figure 1. Sample path of ( $Z ; V)$-values for artificial data $\alpha=0.05, \beta=0.1, \delta=0.2546$ 
As a numerical example, we generated artificial data, which comprised 6 loci, diplotype configuration data. A set of 200 cases and controls were generated $(k=1)$, respectively. Sequential t-test was performed on the artificial data, using the available case and control observations in the matched set. Figure 1 shows the sample path of $(Z ; V)$-values for the controls matched case in an open double sequential t-test with $\alpha=0.05$, power, $1-\beta=0.9$, and $\theta_{R}=0.2546$, where the vertical dashed line indicates the required case-control pairs when fixed test was performed and inclined dashed lines indicate the critical boundaries of the test. In this analysis, the sequential test needed 36 case-control pairs to reject $H_{0}$, whereas the fixed test needed 69 pairs.

We have compared two approaches to perform a t-test with a view to applying such a test in prospective cohort studies for preliminary evaluations of whether or not there is a relevant difference between the case with a disease and disease-free control subjects. Whitehead's procedure is analogous to the SPRT but is based on the profile likelihood rather than a likelihood ratio. We extended the procedure for studies with quantitative data. We have compared the effect of this method with the results of a non-sequential analysis. As we have shown, the use of a sequential t-test to distinguish between promising and less promising hypotheses can save precious biological material. Sequential methods are thus an efficient way to handle the available data.

\section{Concluding Remarks}

Deviations from the HWE can indicate inbreeding, population stratification, and even problems in genotyping. The deviations can also provide evidence for associations, when we use a sample of affected individuals. An association analysis may be regarded as a test for the presence of a difference in allele frequencies between the case and the controls. There is a growing interest in the possibility of conducting genome-wide association studies for a variety of diseases, whereas there is little consensus on methods of design and analysis. Recently, all genome-wide association studies of human populations either have adopted a multistage design or are planning to adopt it. As the necessary sample size increases, however, there still remain problems of adjustment for the genome-wide multiple testing on association studies. We reviewed a number of different software packages designed to infer haplotypes, as well as for identifying htSNPs. We point out that the sequential statistical methods can provide researchers with a possible timing for terminating an investigation. This timing can be defined as the time when sufficient evidence has been accumulated to accept the null hypothesis or to reject it in favor of the alternative hypothesis. In conclusion, the sequential test requires, on average, fewer observations to make a decision than the corresponding fixed sample size test.

\section{References}

[1] Amos CI, Page G, Cost of linkage versus association methods, In: Rao DC, Province MA(eds) Genetic dissection of complex traits, Academic, San Diego, 231-221 (2001).

[2] Armitage P, Tests for Linear Trends in Proportions and Frequencies, Biometrics, 3, 375-386 (1955).

[3] Breslow NE, Day NE, Statistical methods in cancer research Vol. 1, International Agency for Research on Cancer, Lyon (1980).

[4] Bross I, Misclassification in 2 x 2 tables, Biometrics, 10, 478-486 (1954).

[5] Chen TT, Log-linear models for categorical data with misclassification and double sampling, $J$. 
Am. Stat. Assoc., 74, 481-488 (1979).

[6] Cochran WG, Errors of measurement in statistics, Technometrics, 10, 637-666 (1968).

[7] Collins FS, Guyer MS, Charkravati A, Variations on a theme: cataloging human DNA sequence variation, Science, 278, 1580-1581 (1997).

[8] Crow JF, Eighty years ago: the beginnings of population genetics, Genetics, 119, 473-476 (1988).

[9] Devlin B, Roeder K, Genomic control for association studies, Biometrics, 55, 997-1004 (1999).

[10] Feder JN, Gnirke A, Thomas W, Tsuchihashi Z, Ruddy DA, Basava A, Dormishian F et al. A novel MHC Class I like gene is mutated in patients with hereditary haemochromatosis, Nat. Gen., 13, 399-408 (1996).

[11] Gabriel SB, Schaffner SF, Hguyen H, Moore JM, Roy J, Blumenstiel B, Higgins J et al, The structure of haplotype blocks in the human genome, Science, 296, 2225-2229 (2002).

[12] Goodman SN, Berlin JA, The Use of Predicted Confidence Intervals When Panning Experiments and the Misuse of Power When Interpreting Results, Annals of Internal Medicine, 121, 200-206 (1994).

[13] Gordon D, Finch SJ, Nothnagel M, Ott J, Power and sample size calculation for case-control genetic association tests when errors are present: application to single nucleotide polymorphisms, Hum. Hered., 54, 22-33 (2002).

[14] Gordon D, Levenstien MA, Finch SJ, Ott J, Errors and linkage disequilibrium interact multiplicatively when computing sample sizes for genetic case-control association studies, Pac. Symp. Biocomput., 8, 490-501 (2003).

[15] Gordon D, Ott J, Assessment and management of single nucleotide polymorphism genotype errors in genetic association analysis, Pac. Symp. Biocomput., 6, 18-29 (2001).

[16] Gu C, Rao DC, Optimum study designs, In: Rao DC, Province MA(eds) Genetic dissection of complex traits, Academic. San Diego, 439-457 (2001).

[17] Gustafson P, Le ND, Saskin R, Case-control analysis with partial knowledge of exposure misclassification probabilities, Biometrics, 57, 598-609 (2001).

[18] Hardane JBS, An exact test for randomness of mating, J. Genet., 52, 631-635 (1954).

[19] Hardy HG, Mendelian proportions in a mixed population, Science, 28, 49-50 (1908).

[20] Hernandez JL, Weir BS, A disequilibrium coefficient approach to Hardy-Weinberg equilibrium testing, Biometrics, 45, 53-70 (1989).

[21] Hochiberg, Use of double sampling schemes in analyzing categorical data with misclassification errors, J. Am. Stat. Assoc., 72, 914-921 (1977).

[22] Hoenig JM, Heisey DM, The Abuse of Power: The Pervasive Fallacy of Power Calculations for Data Analysis, The American Statistician, 55, 19-24 (2002).

[23] Janis EW, David JC, Goncalo RA, A note on exact test of Hardy-Weinberg equilibrium, Am. J. Hum. Genet., 76, 887-893 (2005).

[24] Jiang R, Dong J, Wang D, Sun FZ, Fine-scale mapping using Hardy-Weinberg disequilibrium, Ann. Hum. Gen., 65, 207-219 (2001).

[25] Kruglyak L, Prospects for whole-genome linkage disequilibrium mapping of common disease genes, Nat.Genet., 22, 139-144 (1999).

[26] Lavene H, On a matching problems arising in genetics,Ann. Math. Stat., 20, 91-94 (1949).

[27] Mitra SK, On the limiting power function of the frequency chi-square test, Ann. Math. Stat., 29, 1221-1233 (1958).

[28] Monte VL. Anderson RL, An investigation of the effect of misclassification on the properties of $\chi^{2}$-test in the analysis of categorical data, Biometrika, 52, 95-109 (1965).

[29] Nielsen DM, Ehm MG, Weir BS, Detecting marker-disease associations by testing for 
Hardy-Weinberg disequilibrium at a marker locus, Am. J. Hum. Gen., 63, 1531-1540 (1998).

[30] Ohashi J, Tokunaga K, The power of genome-wide association studies in complex disease genes: statistical limitations of indirect approaches using SNP markers, J. Hum. Genet., 46, 478-482 (2001).

[31] Rice KM, Holmans P, Allowing for genotyping error in analysis of unmatched case-control studies, Ann. Hum. Genet., 67, 165-174 (2003).

[32] Risch N, Searching for genetic determinants in the new millennium, Nature, 405, 847-856 (2000).

[33] Risch N, Merikangas K, The future of genetic studies of complex human diseases, Science, 273, 1516-1517 (1996).

[34] Sasieni PD, From Genotypes to Genes: Doubling the Sample Size, Biometrics, 53, 1253-1261 (1997).

[35] Song K, Elston RC, A powerful method of combining measures of association and Hardy-Weinberg disequilibrium for fine-mapping in case-control studies, Statistics in Medicine, 25, 105-126 (2005).

[36] Suchard MA, Bailey JN, Elashoff DA, Sinsheimer JS, SNPing away at candidate genes, Genet. Epidemiol., suppl 1, S643-648 (2001).

[37] Tenenbein A, A double sampling scheme for estimating from binomial data with misclassifications, J. Am. Stat. Assoc., 65, 1350-1361 (1970).

[38] Tenenbein A, A double sampling scheme for estimating from misclassified multinomial data with application to sampling inspection, Technometrics, 14, 187-202 (1972).

[39] van den Oord EJ, Sullivan PF, A framework for controlling false discovery rates and minimizing the amount of genotyping in the search for disease mutations, Hum. Hered., 56, 188-199 (2003).

[40] Wald A, Sequential analysis, New York: John Wiley (1947).

[41] Weinberg W, On the demonstration of heredity in man, In Boyer SH, trans (1963) Papers on human genetics, Prentice Hall, Englewood Cliffs, NJ (1908).

[42] Wellek S, Test for establishing compatibility of an observed genotype distribution with Hardy-Weinberg equilibrium in the case of a biallelic locus, Biometrics, 60, 694-703 (2004).

[43] Whitehead J, The design and analysis of sequential clinical trials, 2nd ed. Chicester: Ellis Horwood Limited (1992).

[44] Shephard N, Guide to Genetic Analysis, Center for Integrated Genomic Medical Research (2006). (URL=http://slack.ser.man.ac.uk/) 